\title{
Spinal Liquoral Metastasis from Cerebral Anaplastic Oligodendroglioma: Case Report
}

\author{
Fraioli MF, Pagano A, laquinandi A, Fraioli B and Lunardi P \\ ${ }^{1}$ Department of Neurosurgery, University of Rome Tor Vergata, Rome, Italy \\ ${ }^{2}$ Department of Radiotherapy, CIRAD Villa Benedetta, Rome, Italy
}

Corresponding author: Fraioli Mario Francesco, Department of Neurosurgery, University of Rome Tor Vergata, Via Oxford 81, 00133 Rome, Italy, Tel: +39-06-20903056, Fax+39-06-20903056; E-mail: mff77@libero.it

Received: Jul 12, 2016; Accepted: Jul 27, 2016; Published: Jul 29, 2016

Citation: Fraioli MF, Pagano A, laquinandi A, et al. Spinal Liquoral Metastasis from Cerebral Anaplastic Oligodendroglioma: Case Report. J Neurol Neurosci. 2016, 7: S3.

\begin{abstract}
Background: Spinal cord metastasis is an uncommon event in glioblastoma, symptomatic lesions are very rare, and this occurrence in anaplastic oligodendroglioma is exceptional. Only 16 cases of symptomatic spinal cord metastasis from brain oligodendroglioma have been reported in the literature.
\end{abstract}

Case report: We present the case of a 42 years old woman with multiple symptomatic spinal cord metastasis discovered one year after surgical removal and postoperative radiotherapy for a right fronto-temporal anaplastic oligodendroglioma (grade III WHO).

Conclusion: Although spinal cord metastasis from brain anaplastic oligodendroglioma is very rare, in our opinion it could be useful to perform screening spine MRIs to early detect the eventual diffusion of the oncologic disease. Differently, spinal MRI should be mandatory in all patients in whom an eventual reoperation could be proposed, because the detection of spinal localizations indicates a rapid poor prognosis and an eventual reoperation could be useless in terms of prolonged survival time.

Keywords: Anaplastic oligodendroglioma; Spinal cord metastasis; Screening spinal MRI

\section{Introduction}

Oligodendrogliomas represent $2-4 \%$ of primary brain tumours [1] and anaplastic oligodendroglioma (grade III WHO) is $20 \%$ to $51 \%$ [2] of them. Spinal intradural metastasis is an uncommon event [3] in glioblastoma, symptomatic lesions are very rare, and this occurrence in anaplastic oligodendroglioma is exceptional. To our knowledge, only 16 cases of symptomatic spinal cord metastasis from brain oligodendroglioma have been reported in the literature $[1,4]$. Not rarely, when a high grade glioma recurs, a new surgical treatment is often object of discussion especially if patient's age is less than 60 years. Therefore, it is strictly necessary to evaluate the utility of repeated surgery on prognosis, and its possible side effects and deficit; generally, it is important to avoid quality worsening of the remaining life.

\section{Case Report}

Forty-two years old woman treated 25 years previously in another Institute for a right parietal lobe arterovenous malformation by stereotactic radiosurgery (40 Gy). In December 2008 the patient accessed to emergency unit of another hospital presenting a left arm and facial sensitive seizure, followed by transient impairment of consciousness with maintenance of motor automatisms. Brain MRI showed a small lesion in the deep right temporal lobe with insula involvement, characterized by hyperintensity in FLAIR weighted sequence (Figure 1A), without enhancement after administration of contrast medium. Spectroscopy was aspecific, without alterations of Cho/Cre ratio. The lesion was interpreted as a low grade glioma or, in second instance, as gliosis related to the previous irradiation, and she was discharged with antiepileptic medications and the indication to perform a control MRI after 4 months.

However, the patient didn't perform any control and presented to our hospital in 2010, July, for a generalized seizure; MRI showed a considerable growth of the previously detected lesion, which presented hyperintense in T2 flair images with some nodular centimetric areas enhancing after gadolinium (Figures 1B and 1C). The patient was operated on by a right fronto-temporal craniotomy and a gross total removal was made; tumoral tissue strictly adjacent to the middle cerebral artery vessels of sylvian fissure was left in situ. The histological examination reported anaplastic oligodendroglioma (grade III WHO). 

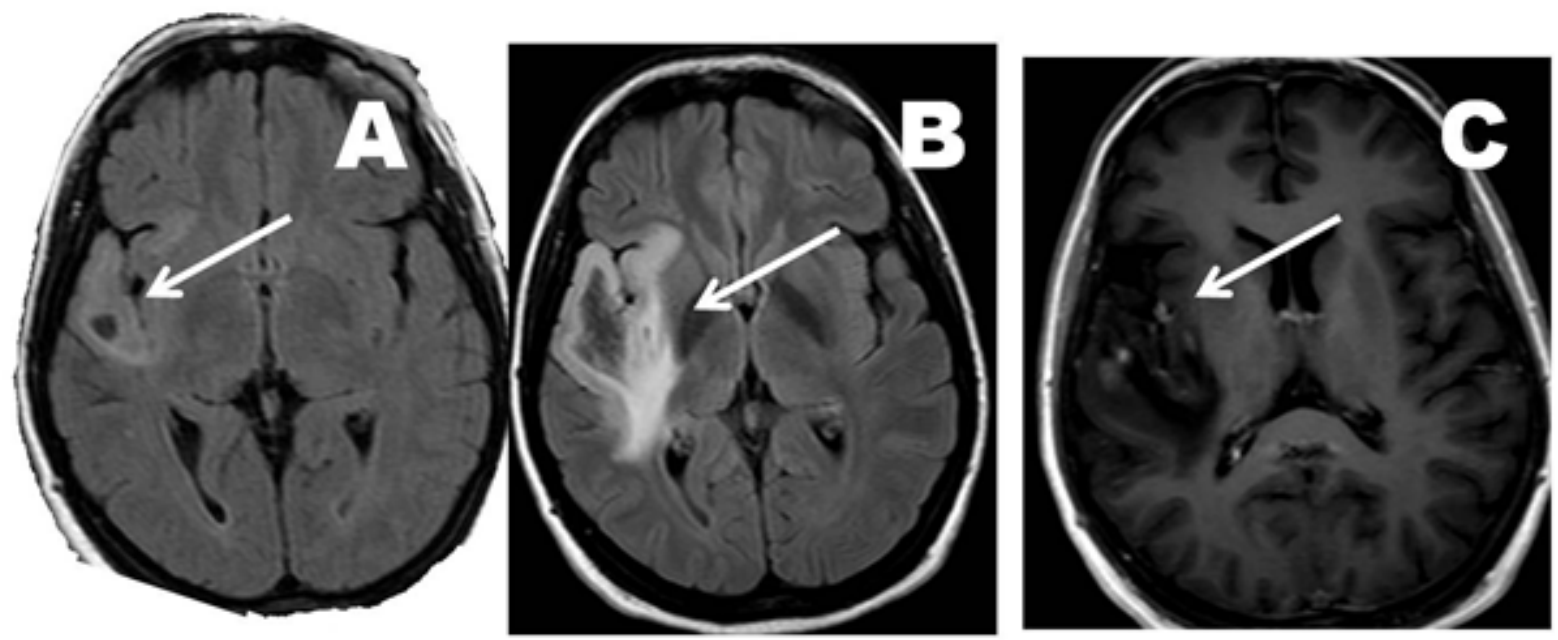

Figure 1 (A) T2 Flair weighted MRI performed in 2008 which shows a small hyperintense right temporal area (arrow). (B) T2 Flair weighted MRI performed in 2010 which shows an enlargement of the above mentioned alteration of signal, with extension to right frontal lobe (arrow). (C) Post-contrast T1 weighted MRI performed in 2010 (the same of Figure 1B) which shows contrast enhancement of some nodular centimetric areas.

After 20 days from surgery, the patient was submitted to postoperative radiotherapy; a total dose of 60 Gy was delivered on the surgical cave and on the residual tumour. In 2011, August, the patient came back to our Department for a progressive drug-resistant cervical pain, started 15 days before. A total spine MRI showed multiple intradural subcentimetric lesions at cervical, thoracic and lumbar spine enhancing after gadolinium (Figure 2). Moreover, a brain MRI showed local tumour recurrence with the typical characteristics of high grade glioma, involving also deep areas as internal capsule and corona radiata. The liquor examination obtained by lumbar rachicentesis showed presence of neoplastic cells typical of the anaplastic oligodendroglioma. On the basis of these findings, the patient was not submitted to cerebral reoperation, although a large part of recurrent tumour was not localized in eloquent areas.
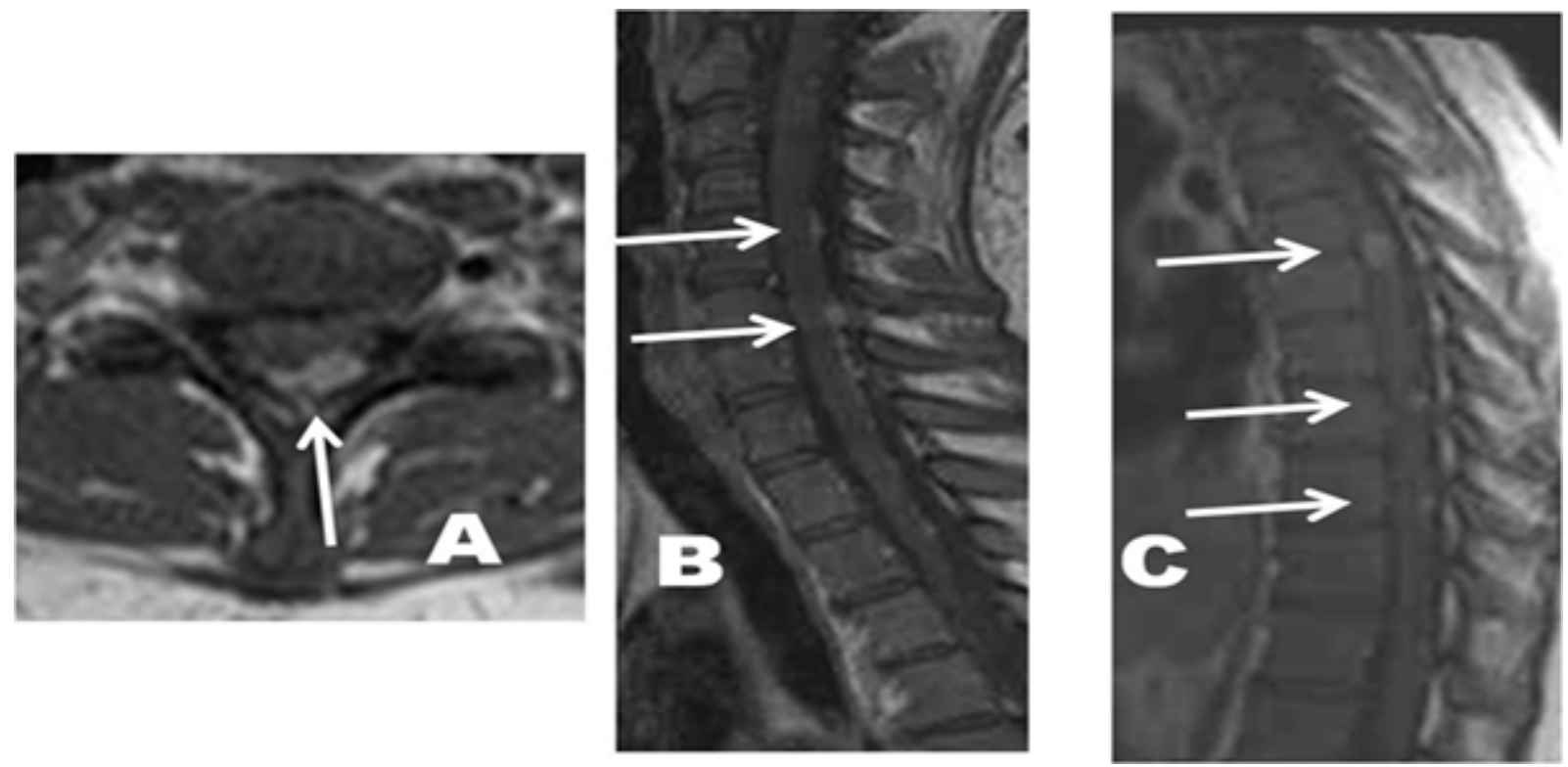

Figure 2 (A) post-contrast T1 axial MRI of cervical spine which shows a hyperintense intradural lesion adjacent to the posterior part of the spinal cord (arrow). (B) post-contrast T1 sagittal MRI of cervical spine which shows multilevel hyperintense intradural lesions adjacent to the posterior part of the spinal cord (arrows). (C) post-contrast T1 sagittal MRI of dorsal spine which shows multilevel hyperintense intradural lesions adjacent to the spinal cord (arrows). 


\section{Discussion}

Spinal metastasis from cerebral glioma were described for the first time in 1926 by Bailey and Cushing [5].

At present, incidence of the clinical cases seems to increase. The increased availability of radiologic examinations raises the diagnosis of the spinal asymptomatic localizations. Moreover, the evolution of surgical techniques (neuronavigator, intraoperative MRI) and postoperative therapies (radiotherapy and chemotherapy) determined an extension of survival time of patients affected by cerebral glioma, with consequent increasing rate of spinal tumour diffusion which represents an evolution of the oncologic disease [6].

The rarity of spinal intradural metastasis could be identified in [7] the presence of the blood-brain barrier; the absence of a brain lymphatic drainage; the short survival time of the patients affected by anaplastic oligodendroglioma. In our review we found several clinical cases of metastasis from anaplastic brain oligodendroglioma and the interested districts were lymphonodes, bones [8], epidural spinal space [9] and spinal cord [10]. The likelihood that anaplastic oligodendroglioma can spread into the subarachnoid space by the liquor is an event still more rare and not correlated with the grade of anaplasia, in the opinion of some authors [3].

Features that seem to facilitate liquoral spreading can be identified in the ventricular extension of the tumor, tumor localized in proximity of the ventricle, surgical treatment and the handling of the tumor, multiple surgical treatments [3].

To our knowledge, only 16 cases of symptomatic spinal cord metastases from cerebral oligodendroglioma were reported in the literature [4]; in all these cases, spinal liquoral metastasis appeared after surgical treatment. The interval between surgery and symptoms of liquoral metastasis is usually 3 months - 6 years [1], differently from our patient who presented symptomatic spinal lesions one year after surgery.

Because of the rarity of spinal cord metastasis from gliomas, a standardized treatment does not exist. In particular, surgery is not indicated because spinal metastasis are usually multiple at the moment of diagnosis. Both systemic and local chemotherapy is ineffective. Radiotherapy represents the best treatment determining a reduction of the pain, but without improvement of the neurological deficits [7] and the survival time.

The increasing detection of spinal metastasis also from anaplastic oligodendroglioma, in our opinion, is important: when a recurrent glioma is localized in a non-eloquent brain area, for example in the right anterior frontal lobe as in our reported case, some neurosugeons could propose a reoperation followed by adjuvant therapies; in our opinion, before make this decision, performing a total spine postcontrast MRI should be useful also for anaplastic oligodendroglioma, because the detection of spinal metastasis is possible and this aspect suggests poor prognosis with short survival time and surgical reoperation could be useless in terms of prolonged survival time.

\section{Conclusion}

Spinal liquoral metastasis from cerebral anaplastic (grade III WHO) oligodendroglioma represent a very uncommon event. In our review we found only 16 cases of symptomatic spinal liquoral metastasis, therefore a standardized treatment does not exist. In our opinion, it could be useful to perform a screening total spine MRI in all patients operated for high grade gliomas to early detect spinal metastasis and therefore to understand the stadium of the disease; differently, spinal MRI should be mandatory in all patients affected by spinal pain in whom a reoperation could be proposed.

\section{References}

1. Pinar AO, Ilkay I, Hakan O, Figen S, Osman ekin O (2008) Unusual massive spinal metastases of an intracranial oligodendroglioma. Turkish Neurosurgery 8: 276-280.

2. Nijjar TS, Simpson WJ, Gadalla T, McCartney M (1993) Oligodendroglioma: The Princess Margaret Hospital Experience (1958-1984). Cancer 71: 4002-4006.

3. Reggiani R, Solimè F, Del Vivo RE, Nizzoli V (1971) Intracerebral oligodendroglioma with metastatic involvement of the spinal cord. J Neurosurg 35.

4. Elefante A, Peca C, De Caro MDB, Russo C, Formicola F, et al. (2012) Symptomatic spinal cord metastasis from cerebral oligodendroglioma. Neurological Sciences 33: 609-613.

5. Bailey $P$, Cushing $H$ (1926) Tumors of the glioma group, JP Lippincott, Philadelphia.

6. Hubner F, Braun V, Richter HP (2001) Case report of symptomatic metastases in four patient with primary intracranial gliomas. Acta Neurochir 143: 25-29.

7. Zustovich F, Della PA, Scienza R, Anselmi P, Furlan C, et al. (2008) Metastatic oligodendrogliomas: a review of the literature and case report. Acta Neurochir (Wien) 150: 699-702.

8. Macdonald DR, O' Brien RA, Gilbert JJ, Cairncross JG (1989) Metastatic anaplastic oligodendroglioma. Neurology 39: 1593-1569.

9. Kim JG, Park CO, Hyun DK, Ha YS (2003) Spinal epidural metastases of cerebral oligodendroglioma. Yonsei Med J 44: 340-346.

10. Van Velthoven V, Calliuw L, Caemaert J (1988) Intramedullary spread of a cerebral oligodendroglioma. Surg Neurol 30: 476-481. 